\title{
BMJ Open Lung cancer treatment rates and the role of the lung cancer nurse specialist: a qualitative study
}

\author{
Angela Mary Tod, ${ }^{1}$ Judy Redman, ${ }^{2}$ Ann McDonnell, ${ }^{2}$ Diana Borthwick, ${ }^{3}$ \\ John White ${ }^{4}$
}

To cite: Tod AM, Redman J, McDonnell A, et al. Lung cancer treatment rates and the role of the lung cancer nurse specialist: a qualitative study. BMJ Open 2015;5: e008587. doi:10.1136/ bmjopen-2015-008587

- Prepublication history for this paper is available online. To view these files please visit the journal online (http://dx.doi.org/10.1136/ bmjopen-2015-008587).

Received 23 April 2015 Revised 1 August 2015 Accepted 25 August 2015

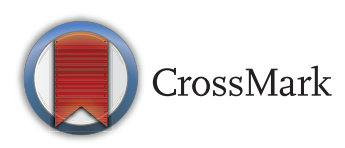

${ }^{1}$ School of Nursing and Midwifery, University of Sheffield, Sheffield, UK ${ }^{2}$ Centre for Health and Social Care Research, Sheffield Hallam University, Sheffield, England

${ }^{3}$ Edinburgh Cancer Centre, Western General Hospital, Edinburgh, Scotland ${ }^{4}$ St James's Institute of Oncology, Leeds Teaching Hospitals NHS Trust, Leeds, England

Correspondence to Professor Angela Mary; a.tod@sheffield.ac.uk

\section{ABSTRACT}

Objectives: This qualitative study examines how the Lung Cancer Nurse Specialist (LCNS) role operates and why they may be able to increase access to treatment.

Setting: 4 Hospital NHS Foundation Trusts in England.

Design: A multiple case study design using semistructured interviews, observation and Framework Analysis techniques.

Participants: Four LCNSs, comprised the 'cases'. Twenty four clinicians who worked with the LCNS participated in individual interviews. Six LCNSs took part in a group interview and 60 lung cancer multidisciplinary team (MDT) members and coordinators were observed in the MDT meeting.

Results: The LCNS is crucial within the MDT and can act as a catalyst to patient access to treatment. The study identified the clinical activity (assessment, managing symptoms, psychological support and information provision) and role characteristics that can facilitate treatment access. These characteristics are the LCNS's presence across the patient pathway, acting as the 'hub' of the MDT, maintaining a holistic patient focus and working to an advanced level of practice. The findings indicate how factors may have a cumulative impact on treatment access.

Conclusions: If UK patient with lung cancer survival rates are to improve in line with comparable countries, we need to employ every advantage. This study demonstrates how the LCNS role may open doors to positive patient outcomes, including treatment. Further research is required to explore patients' experiences, decision-making and attitudes to treatment.

\section{INTRODUCTION}

Lung cancer is the second most commonly diagnosed cancer in the UK and the most common cause of cancer death. ${ }^{1}$ In 2011, 43463 people were diagnosed and 35184 people died from lung cancer. ${ }^{1}$ UK Lung Cancer survival rates are lower than those in comparable European countries and other countries including Canada and Australia. ${ }^{2}$ Improving anticancer treatment rates

\section{Strengths and limitations of this study}

- The study was successful in generating in-depth insight into how the Lung Cancer Nurse Specialists (LCNSs) conducted their work, and why they may be able to increase access to treatment. The clinical activity and role characteristics that contributed to this impact were identified.

- Data were collected from individual and group interviews, observation of multidisciplinary team meetings and selected documents (eg, job descriptions, patient pathways and protocols). This triangulation adds strength to and helps to verify the findings.

- This study focused on capturing staff experiences and perceptions. Further research is required that explores patients' experiences, decision-making and attitudes to treatment.

- The study was small and limited to four case studies but included a range of sites in terms of delivering chemotherapy or not, and how the LCNS role operated. This range, along with the resonance of findings with other research, adds to the transferability of the findings.

- Specialist surgical and radiological centres were excluded. Our aim was to observe and understand how the LCNS worked in peripheral referring Trusts which form the majority of services.

(surgery, chemotherapy and radiotherapy) is a key instrument in improving lung cancer survival. $^{3}$

The National Lung Cancer Audit (NLCA) was established in 2004 to monitor and measure outcomes and quality of care against fixed standards. ${ }^{3}$ NLCA reports indicate UK lung cancer survival rates are improving in line with increased access to treatment. For 2012, the anticancer treatment rate and the overall surgical treatment rate for England and Wales both increased by almost one percentage point to $60.8 \%$ and $15.2 \%$ respectively. ${ }^{3}$ The number of people with lung cancer surviving to 1 year has almost doubled in the past 20 years. This progress is explained by improvements to 
treatment rates as well as earlier diagnosis and organisational developments. ${ }^{1} \quad 4 \quad 5$ However, concern remains regarding regional variations in treatment rates across the UK, that cannot be explained by case-mix or data quality. ${ }^{3} 6$

For the 2010 and 2011 audit years the NLCA reported an association between having access to a Lung Cancer Nurse Specialist (LCNS) and receiving anticancer treatment. For 2010/2011 audit years, 64\%/64.3\% of patients seen by a LCNS received anticancer treatment, compared to $30 \% / 28.7 \%$ of those who did not see a nurse specialist. ${ }^{6-11}$ While this finding does not demonstrate a causal link, the association between access to a nurse specialist and anticancer treatment rates was not explained by age, disease stage or performance status. ${ }^{6}$ In its editorial reporting this finding, The Lancet commented that increased access to treatment is one of many benefits from nurse specialist care. ${ }^{8}$ Further analysis of the 2010 NLCA data, adjusting for case-mix, indicated an association between being seen by a LCNS and increased rates of active treatment, particularly chemotherapy or radiotherapy, but not surgery. ${ }^{9}$

Many studies (mainly surveys) have examined the role and value of the LCNS and the nurse specialist impact on care quality, patient outcomes and cost of services. This evidence indicates that benefits are accrued though the LCNS working collaboratively within the multidisciplinary team (MDT) to deliver proactive care management across the patient pathways. They provide advanced clinical practice, accomplished communication and complex navigation and brokering for patients. ${ }^{12-17}$ However, no study has been conducted to specifically examine why or how the LCNS may have an impact on increasing anticancer treatment rates.

The study presented here used qualitative methods to generate in-depth understanding of what aspects of the LCNS role may contribute to increased treatment rates. It is recognised that other factors will influence treatment rates, for example if the LCNS works in a specialist treatment centre or a peripheral centre, or if the LCNS is a single practitioner or has other specialist nurse colleagues. These explanations are acknowledged but are not the focus of this study. The aim was to examine the nature of the LCNS role and understand how it is operationalised, with the patient and MDT, to facilitate access to treatment.

\section{METHODS}

\section{Design}

A multiple case study design ${ }^{18}{ }^{19}$ was used to develop detailed insight and understanding of LCNS work in four case study sites. The sites were all National Health Service (NHS) Foundation Trusts, but varied in terms of size, geographical location and demographics (serving rural and urban populations). The focus was on what aspects of the LCNS work across the four different sites may help to increase treatment access.
NHS Research Ethics approval was not required as only staff were involved.

\section{Sample}

Purposive sampling was used to identify four case study sites. ${ }^{20}$ Using the National Lung Cancer Audit (NLCA) we recruited a mixture of services with reported high and low access to treatment and to the LCNS. A project advisory group that included members of the National Lung Cancer Forum for Nurses (NLCFN)-guided sampling decisions. There were four groups of participants:

1. Each 'case' was a LCNS working in a lung cancer service in an NHS Foundation Trust. Four 'cases' were purposively selected to reflect site variation in access to treatment and LCNS (see table 1). ${ }^{20}$ Specialist surgical and radiology centres were excluded. Our focus was on how the LCNS role operated in peripheral sites which form the majority of services rather than specialist sites. In addition an assumption was made that specialist sites may not have the same barriers to treatment access as peripheral referring centres, since treatment services are on site. Therefore, location of a specialist service is likely to increase access in itself. For example, patients first seen in an MDT based in a thoracic surgical centre are more likely to receive surgery than those seen in peripheral centres. ${ }^{21}$ We wanted to observe how LCNSs worked to help patients access treatment where such advantages did not exist. We did seek a mix in terms of chemotherapy delivery across the four sites.

2. Individual interviews were conducted with up to six clinicians per case. In addition to the LCNS $(n=4)$ this sample comprised the lead clinician for the MDT $(n=4)$, plus three to five other MDT members or clinical colleagues $(n=16)$. A total of 24 participants took part in individual interviews (table 2).

3. Structured observations were conducted of the MDT meetings in each of the case study sites to examine group interaction and treatment decision-making. Between 12 and 17 clinical staff attended each MDT meeting, plus the MDT co-ordinator (total $n=60$ ). Between two and three key documents were also analysed for each site, including the LCNS job descriptions and patient pathways.

4. A group interview was conducted to expand and test emerging findings from the case studies with LCNSs from different Trusts, recruited from the NLCFN membership ( $\mathrm{n}=6)$.

\section{Recruitment}

The four LCNSs who were the cases at each site were contacted and recruited by members of the NLCFN Executive Committee who outlined the study. With their agreement, the research team were informed and contacted the LCNS to discuss participation, arrange an initial visit and obtain consent. The LCNSs were asked 
Table 1 Characteristics of case study sites

\begin{tabular}{|c|c|c|c|c|c|c|c|}
\hline \multirow[b]{2}{*}{ Site } & \multirow{2}{*}{$\begin{array}{l}\text { Year in } \\
\text { post }\end{array}$} & \multirow{2}{*}{$\begin{array}{l}\text { LCNS } \\
\text { number } \\
\text { on site }\end{array}$} & \multirow{2}{*}{$\begin{array}{l}\text { Access to } \\
\text { treatment/ } \\
\text { LCNS } \\
\text { access* }\end{array}$} & \multicolumn{3}{|c|}{ Active antilung cancer treatment on site } & \multirow[b]{2}{*}{ Focus of LCNS } \\
\hline & & & & Surgery & Radiotherapy & Chemotherapy & \\
\hline$A$ & 2007 & 2 & High/high & No & No & Yes & $\begin{array}{l}\text { Sees patients in outpatient clinic. } \\
\text { Mostly see patients in community } \\
\text { including postdiagnosis home } \\
\text { visits. A lot of telephone } \\
\text { consultations }\end{array}$ \\
\hline B & 2002 & 2 & $\begin{array}{l}\text { High/ } \\
\text { low-mid }\end{array}$ & No & No & No & $\begin{array}{l}\text { One stop outpatient clinic and } \\
\text { respiratory clinic. Does home and } \\
\text { ward visits. Work with patients } \\
\text { postdiagnosis and now setting up } \\
\text { rehabilitation service }\end{array}$ \\
\hline $\mathrm{C}$ & 2000 & 1 & Low/high & No & No & $\begin{array}{l}\text { Yes-regimen } \\
\text { dependent; some } \\
\text { referred to tertiary } \\
\text { centre }\end{array}$ & $\begin{array}{l}\text { Sees patients on wards and in } \\
\text { outpatient clinic (including } \\
\text { preoperative, bronchoscopy } \\
\text { clinics) and chemotherapy unit. } \\
\text { Some home visits }\end{array}$ \\
\hline $\mathrm{D}$ & 2010 & 2 & Low/low & No & No & $\begin{array}{l}\text { Yes-regimen } \\
\text { dependent. Some on } \\
\text { other trust site }\end{array}$ & $\begin{array}{l}\text { Cover oncology clinics. See } \\
\text { patients on ward but focus is on } \\
\text { telephone and clinic work }\end{array}$ \\
\hline
\end{tabular}

to approach the lead clinician, and other MDT members and clinical colleagues with insight into their role to discuss study participation. The potential participant then met with a researcher for further discussion and interview. The LCNS was also asked to broker agreement of the MDT for the research team to conduct the observation. The group interview participants were recruited through the NLCFN Executive Committee. Written informed consent was obtained from all interview and observation participants.

\section{Data collection}

Data were collected between March and September 2013. Each case study consisted of three stages detailed in box 1. Individual interviews were conducted in the participant's workplace where possible, otherwise by telephone. The group interview with NLCFN members was conducted face to face. Data collection was guided by the use of individual and group interview schedules, observation and document templates and pro forma that had been developed through consideration of relevant literature and informed by discussion with the NLCFN reference group. Interviews and observations were conducted by three of the authors (AMT, JR and $\mathrm{AM})$.

Interviews were audio recorded and transcribed. Two researchers conducted each observation and independently made detailed contemporaneous notes which were cross-referenced later and checked for accuracy. Field notes were taken for interviews and observations. Interview transcripts and field notes were all anonymised by removing identifiable data about sites and participants. The data were transferred to $q s r$ NVIVO (V.10) for analysis.

Table 2 Participants interviewed in case study sites

\begin{tabular}{lll}
\hline $\begin{array}{l}\text { Case study } \\
\text { site }\end{array}$ & Participants \\
\hline A & $\begin{array}{l}\text { LCNS (Case), Consultant Respiratory Physician, Consultant Clinical Oncologist, Consultant Thoracic } \\
\text { Surgeon, Specialist Registrar in Respiratory Medicine }\end{array}$ \\
B & $\begin{array}{l}\text { LCNS (Case), LCNS 2, Consultant Respiratory Physicians×2, Consultant Clinical Oncologist, Secretary/ } \\
\text { Lung MDT Coordinator, Clinical Nurse Manager }\end{array}$ \\
C & LCNS (Case), Consultant Respiratory Physician, Consultant Clinical Oncologist, Consultant in Palliative & 6 \\
D & Medicine Cancer Pathway Coordinator, Clinical Nurse Manager & \\
& LCNS (Case), LCNS 2, Consultant Respiratory Physician, Consultant in Palliative Medicine, MDT
\end{tabular}


Box 1 Stages of data collection in the four case study sites

Stage 1: An initial set up visit to discuss involvement, obtain consent from the Lung Cancer Nurse Specialist (LCNS), arrange the practicalities of the case study visit and conduct initial interview.

- Stage 2: A case study visit including individual interviews and an MDT meeting observation.

- Stage 3: A follow-up interview with the LCNS to feed back to the site and clarify any points. (In two cases, this was conducted by telephone by negotiation with the LCNS).

An observation was conducted of the MDT meeting in each of the four sites. Two of the research team attended the meeting following obtaining signed consent from each person attending. The researchers sat at the back or to the side of the room and recorded on a pro forma brief notes describing the treatment decision-making process for each patient, for example, who was involved, what was taken into account and the decision made. At the end of the meeting fieldnotes were written to provide a brief description of the meeting (eg, length, who participated, who dominated decisions, was everyone listened to, were opinions sought when not volunteered) as well as key issues identified. In one of the observations a LCNS was not present due to sickness. This was the last observation. Comparing the observation where a LCNS was absent, to the other three MDT observations provided useful insight and helped clarify how the LCNS contributed to decisions when present.

\section{Data analysis}

Framework analysis was used to interpret the data and identify key themes and issues to explain the contribution of the LCNS to anticancer treatment access. ${ }^{22} 23$ Framework Analysis is a pragmatic approach used in policy research. Framework allows the integration of preexisting themes and emerging findings into the analysis and provides a clearly defined analytical structure that contributes to the transparency and validity of the results. Five analysis techniques (familiarisation, developing a thematic framework, indexing, charting and mapping and interpretation) were used to identify crosscutting themes shared across case studies. All transcripts were coded independently by one of three researchers (AMT, JR, AM) then coded by another analyst to verify interpretation. Preliminary findings and thematic frameworks were discussed at analysis meetings with the remaining authors (DB and JW) to generate agreement and enhance validity.

\section{RESULTS}

\section{Overview}

The findings contribute significantly to our understanding of how the LCNS has an impact on treatment. The
LCNS worked differently in the different sites according to local resources, geography and demographics. This paper concentrates on the results that explain clinical elements of the LCNS work that may increase treatment access and explain why LCNS activity has this impact. Findings are presented in two sections. The first section acknowledges that much of the clinical activity may not be unique to the LCNS. Aspects of the work will be conducted by other MDT members. The findings clarify the distinctive characteristics of the LCNS role that mean their clinical activity may impact on treatment access. Finally the LCSN clinical activity that contributed to increased treatment access is identified. Key findings are presented here. Illustrative quotes that help to demonstrate the characteristics and activity of the LCNS role are provided in boxes 2 and 3 .

\section{Characteristics of the LCNS role}

The clinical activity presented below is not uniquely conducted by the LCNS, but is also undertaken by other members of the MDT. However, there were four essential characteristics of the LCNS role that interrelate with their clinical activity and so made an impact on treatment access and care. First, the LCNS was a constant presence, constant across the patient pathway, unlike other MDT members, whose input was more episodic. Second, the LCNS was referred to as the 'hub' of the MDT and was central to care delivery and the smooth running of the MDT. This was partly due to their continuous presence across the pathway, but also ascribed to their skills in communication, navigation and brokering between and across patient, professional, service and organisational boundaries. Third, the LCNS was seen as best placed to see the illness in the context of the patient's whole life and therefore accurately assess and support them (box 2). This patient focus was often enhanced by seeing patients at different times on the pathway and in different settings (including the patient's home) and with family. Finally, the advanced level at which the LCNS was working made the services more efficient and enabled timely patient access to interventions. While the MDT members did work collaboratively the LCNS was able to make autonomous clinical decisions that did not require validation or checking by senior medical colleagues. The LCNS was an advanced, but also trusted and respected senior MDT member.

From the observations the LCNSs clearly had the respect of all present and was able to use humour and diplomacy to ensure that a great deal of information was considered, while at the same time the meeting moved at a good pace.

The LCNS was seen to contribute unique insight into the patient that other MDT members did not have. Examples included information on social context, about the patient from other parts of the pathway that others could not remember, and what the lung cancer diagnosis means in their life. The LCNSs had understanding of patient and their lives and were able to move the 
Box 2 Characteristics of the Lung Cancer Nurse Specialist (LCNS) role

\section{Constant supportive presence}

"We're the one constant as well in their journey, when you think the amount of consultants we've gone through, when the registrars come in.....you're their constant because actually the consultant they've got their confidence in, they have seen him, but actually you're the one that's always been there". (Case Study 2 LCNS)

"They're supportive of all the patients' journey, and also in the community and they are one of the pillars of strength in that department, where the patients can lean upon and can discuss any sort of thing, so that's quite important for the patient. It makes the whole cancer journey different for the patients." (Case Study 3 Medical Consultant 2)

\section{The LCNS as the hub of the MDT}

"I guess what they are is they are the primary point of contact, aren't they, for

patients and families, as they coordinate and go through their treatment." (Case Study 2 Medical Consultant 2)

"I mean they're definitely the kingpins in the whole process. The nitty gritty they do

I'm not so in detail, but patients always speak highly of them and it's always that they

know they're not just being number crunched through CS3, there's somebody at the

end of the phone who can speak to them. Often doctors aren't the ones they want to

speak to and they've always got the liaison number to phone up to, so it's a feeling of

importance and a feeling of worth and a feeling of not being left alone with a

condition is one of the most important roles." (Case Study 3 Medical Consultant 2)

Knowing the patient context

"They [LCNS] hold the case. So they hold the case in context." (Case Study 2 Medical Consultant 2)

"I imagine it as a theatre; you've got all these people waiting in the wings and they come in and play their part on the stage with the patient, and it may only be for five minutes or it may be for a full scene, and they then depart. But the people that are on the stage permanently are the patient, the carer and the lung CNS." (Case Study 3 LCNS)

"A lot of people will say to her [the LCNS] have you met this patient, what do you think he would do or, and someone will say his performance status is three, and then the LCNS will say yeah but l've seen him this week and that's a lot better than the last time you saw him. So her opinion and what she knows about the patients is vital when they're making the decision, definitely, because she does have a lot of patient contact." (Case Study 4 C1)

\section{Advanced practice}

I think they're extremely knowledgeable in the field of lung cancer, and I think that their purpose is to, well the way I see it they make the whole of the patient's pathway run smoothly. (Case Study 1. Consultant 1)

"Obviously the notion of the management of symptoms is hugely important as well ....I've got case studies that I present about ... how our interventions help improve people's performance status before they see an oncologist has actually allowed them to have treatment." (Focus group)

discussion on treatment in a patient-focused manner. "Whats best for THIS patient," "What shall we do for HER"

The LCNS were observed to influence decisions throughout the MDT but not through speaking out often or loudly. They presented pertinent facts at the right time and used humour off and on to move things forward.

How these characteristics manifest themselves in terms of increasing access to treatment is illustrated by the quotes in box 2 .

\section{LCNS clinical activity}

Across all the data participants agreed on key aspects of the LCNS clinical activity that in their view contributed towards increased treatment access. However, this impact was realised by integrating the above characteristics with the clinical activity.

\section{Assessment}

LCNS assessment of patients was seen to increase access to treatment by taking into account the broader context of the patient's life, and impact of the diagnosis on the patient's psychosocial circumstances. A key aspect was more considered assessment of performance status. Participants reported the LCNS contribution here was highly valued and could, on some occasions, impact on eligibility for treatment. The expertise and knowledge of the LCNS enabled them to use their judgement to assess the right time to inform and discuss treatment possibilities with patients in an accessible way. In addition, LCNS assessment helped to resolve diagnostic confusion. For example the patient focused nature of the assessment conducted by the LCNS enabled them to swiftly treat the condition. This sometimes made a difference between a patient being suitable for anti-lung cancer treatment or not.

we might see a patient in clinic, do the home visit, realise that they actually are quite fatigued, but the fatigue is because they've lost their appetite, so by improving on symptoms, by improving their appetite, which ultimately would improve fatigue, by just introducing a small dose of steroids might bring their fitness level up to a state where they're then able to get anticancer treatment. So again it's about that holistic assessment and understanding the disease as well and knowing what works (CS3 LCNS1) 


\section{Box 3 LCNS clinical activity}

\section{Assessment}

"They're better at sort of assessing functional status, performance status than a lot of people, and quite often they've seen them in their own home. And they quite often can advocate and say look I know you said this person's performance status two, but I saw them a few days ago, yeah, he's out of bed, but, you know, he sits in his chair or he walks back from one room to another, it's like l've got real doubts about his fitness." (Case Study 3 Specialist Registrar)

"Picking people up pre-diagnosis and taking care of their pain and their breathlessness and their money problems and everything that goes around that holistic assessment bit, that's changed dramatically, so we're able to input more and improve for patients"(Case Study 2. LCNS)

"LCNS1 and LCNS2 a week or two later may have been in touch with the patient and they may have made a stonkingly good recovery from their pneumonia or whatever, and then things open up again and they do become maybe fit for anticancer treatment, and so they'll get the patients chivvied along to the relevant clinic so that they can be offered more active treatment." (Case Study 1 Medical Consultant)

\section{Symptom management and optimising function}

"Nurse prescribing gives us more autonomy. Particularly in the community. We're not having to ring up GPs and say look can you do a prescription"? (Case Study 3 LCNS)

"I suppose what we try to constantly encourage is that patients do try and improve their general health, if we can manage symptoms, get them feeling fitter, then there's always is an opportunity to consider treatment if that's an option. So we're very proactive in that." (Case Study 1 LCNS)

“....we might see a patient in clinic, do the home visit, realise that they actually are quite fatigued, but the fatigue is because they've lost their appetite, so by improving on symptoms, by improving their appetite, which ultimately would improve fatigue, by just introducing a small dose of steroids might bring their fitness level up to a state where they're then able to get anticancer treatment. So again it's about that holistic assessment and understanding the disease as well and knowing what works..." (Case Study 3 LCNS)

"Obviously the notion of the management of symptoms is hugely important as well ....l've got case studies that I present about how our interventions help improve people's performance status before they see an oncologist [and this] has actually allowed them to have treatment." (Focus group 1)

\section{Counselling and psychological support}

'I've had patients who've gone for surgery who are very unsure or have been tentative, the fact that they're offered the support following the surgery has been helpful in that decision making.... In the past I have arranged a home visit for a patient who didn't want treatment, just couldn't see the sense in any chemotherapy treatment, because they were just so devastated at the time, but following discussion, again at the patient's home, agreed to treatment and responded well to treatment and lived for another two years at that point". (Case Study 1 LCNS) "their role is pretty pivotal in that, because the consultants will explain right you've got to have this done, but I think the lung cancer nurses are there to allay their fears, to support them through the process which isn't very pleasant. And I think that's quite important to get them through the investigation process, to allow them to be able to have treatment options at the end of it." (Case Study 1 Consultant 1 )

"it's recognising when we phone them up to see how they are, it may well be that they're getting really depressed, that actually the reason why they're not getting out of bed is not because they're poorly, it's because they're really depressed, and they need the support to get themselves back on their feet to be well enough to have the treatment, because obviously even if someone's mentally unwell it would be very difficult to give them treatment because they're not really going to tolerate them very well. So it's managing any sort of psychological problems or symptom management from a distance that gets a patient back into the clinic, that you can re-assess them again and decide actually yeah they are fit enough for treatment. (Case Study 2.LCNS)

\section{Patient and family information provision}

"We often have patients who at first discussion don't want anything doing or they're very adamant against one treatment or another, but given a bit more time to have a chat about what it might entail they're able to inform patients....making sure that they understand what the implications of that decision or what surgery would involve and what the difference between surgery and radiotherapy is in terms of outcomes. So they have a bit more time to be able to spend, so they influence outcomes ". (Case Study 1 Medical Consultant 2)

"You think well actually l've got to take this very slowly with this patient for them to adjust, for them to understand what's going on as well, and appreciate and accept why we're doing things, and ultimately the diagnosis. And I think if you rush people too quickly sometimes it becomes a jumble in their head, and that's when they misunderstand things". (Case Study 3 LCNS1)

"..I can imagine when you first meet the oncologist and you're told your diagnosis and you're told, it must just be like a bombshell. So I could imagine a lot of the information just doesn't go in does it?...So then they're going to go home and they're going to have the questions. Well the first person they're going to ring is the LCNS....Because when they get home and they sit down and it all sinks in, I think that's when the important questions come for most of them, because it must just be an absolute shock for a lot of them." (Case Study 4 Consultant 1)

In the MDT meetings that were observed the LCNS were seen to contribute to the decision-making by presenting their assessment of performance status as well as their assessment of the patients understanding of their diagnosis and the impact of the illness on the patient's life. This assessment was often the most accurate as the LCNS had seen the patient more often and more recently than other MDT members. In the meeting where the LCNS was absent there was some uncertainty on who had last seen the patient, when and what the most recent performance status assessment was. It was evident from the observations that the LCNS was able to see the whole patients experience across the pathway and how they had improved or deteriorated. 
Other MDT members only saw patients for parts of the pathway so lacked the continuity and comparison throughout the patient journey.

\section{Symptom management and optimising function}

Across the patient pathway participants explained that the LCNS supported better management of symptoms such as breathlessness, pain and fatigue, thus improving eligibility for treatment. Swift, accurate prescribing and titration of medication again helped to maximise fitness, reduce symptoms and increase treatment potential. Treatment options are influenced by stage, grade and type of cancer, comorbidities, and the patient's performance status. The LCNS enhanced patients' opportunities for active treatments by ensuring practical interventions to optimise performance status.

At the end of the three MDT observations where the LCNS was present patients were discussed where interventions from the LCNS had helped improve patient anxiety or symptoms and therefore active treatment was discussed. One optimising function of the LCNS observed in the MDT meetings was their knowledge and navigation of systems and services. Examples included discussions where patients were being seen by, required referral to or treatment by other services prior to lung cancer treatment. For example, if the lung cancer was a secondary and treatment was required for the primary first. The LCNS was clearly well respected and deferred to regarding their background knowledge of these services, other hospitals and clinical teams.

\section{Counselling and psychological support}

Provision of ongoing support regarding the emotional, social or financial repercussions of a lung cancer diagnosis was key to the LCNS preparing patients for treatment and increasing eligibility or acceptance. Examples included overcoming fear and fatalism regarding treatment, mediating the impact of family responses or the sometimes prohibitive cost of travel to treatment. The LCNS was reported to identify and tackle emerging mental ill health (eg, anxiety and depression), and provide listening and counselling, to overcome blame and stigma, promote self-esteem and confidence, address fear and denial, and promote coping mechanisms.

In the observations the LCNS was often asked to provide information regarding the psychological status of the patient, and the preferences and priorities of the patient that may influence treatment potential and acceptance.

\section{Patient and family information provision}

The provision of timely, accurate, trusted and appropriate information and advice to patients and carers helped ensure they were equipped to make informed treatment decisions. This was important where they were previously misinformed about treatments and refused because they felt scared, fearful or hopeless regarding treatment. The LCNS was reported to overcome this barrier to treatment by creating time for patients. This gave patients more control and helped to allay fears related to their prognosis. The LCNS had the expertise to judge the speed of information delivery as well as tailoring the content of that information and support. In addition the LCNS provided information, support and advice to patients and carers regarding aspects of lifestyle that also improved fitness and therefore eligibility for treatment. Key examples here were smoking cessation, physical activity, diet and nutrition and hydration.

The LCNSs who were observed in MDT meetings were seen to keep a record of all key decisions. They also recorded and what they were required to do to co-ordinate these after the meeting and inform the patient and family. This meant liaising with the patient and where necessary with MDT members, other clinicians, other hospitals, other Lung or other cancer MDTs and LCNSs. In addition to co-ordination, the LCNS was seen to take a role in explaining and translating information about services and treatments in an understandable and acceptable way to patients and families. These explanations provided part of LCNS contribution to MDT discussions.

\section{DISCUSSION}

\section{Summary of key findings}

Our study has produced qualitative evidence to illustrate the 'ways of working' which provide clear and plausible mechanisms by which LCNSs can increase access to anticancer treatments.

The LCNSs worked differently across sites according to local context, referral pathways to specialist treatment centres, resources and the population demographics, but there were commonalities in terms of how their impact on treatment access was described. It is those commonalities that this study succeeded in identifying.

This study shows the complexity of the LCNS impact through their clinical activity and the characteristics of their role. They worked across different structures and settings, and with a diverse range of disciplines. A key feature of their working practice was to keep the patient at the centre of treatment decision-making. The LCNS role was highly respected and considered core and integral to the MDT by the members interviewed. The LCNS was a crucial member of the MDT and was a catalyst to a patient being seen to be eligible for treatment. This was realised through the tasks the LCNS undertakes, for example, assessment, managing symptoms, psychological support and information provision.

The findings illustrates how the LCNS role has to embrace certain characteristics in order to impact on treatment access. They were a constant supportive presence across the pathway, acted as the 'hub' of the MDT, maintained a holistic patient focus and worked to an advanced level of practice. These characteristics interact with clinical activity to have a cumulative effect on treatment access. For example, seeing patients across the 
pathway enables the nurse specialist to conduct informed assessment of symptoms and performance status and respond accordingly in terms of information provision, support and treatment.

\section{Strengths and weaknesses}

The study was successful in generating in-depth insight into how the LCNSs conducted their work, and why they may be able to increase access to treatment. Articulating cause and effect in a quantifiable sense would be highly problematic for any element of LCNS work, even in the context of rigorously designed experimental studies, as the impact of many advanced nursing roles is inherently hard to capture. ${ }^{16} 24$ Therefore, the intention here was not to provide statistical data on how often this impact was experienced, but to provide explanations to understand how the impact plays out in clinical practice.

This study focused on capturing staff experiences and perceptions. Further research is required that explores in more depth patients' experiences, decision-making and attitudes to treatment.

Data were collected from individual and group interviews, observation of MDT meetings and selected documents (eg, job descriptions, patient pathways and protocols). This triangulation adds strength to and helps to verify the findings. The study was limited to four case studies of LCNSs in NHS Foundation Trusts. This meant experience in smaller Trusts and specialist surgical and radiological centres were excluded, and may limit transferability of findings. However, the range of disciplines and data collection methods increased the rigour of the study. Caution is still required in making generalisable claims from this data without wider testing of the experiences and practice reported here.

As there was only one or two LCNSs at each site there was potential for participants to feel that they are making a direct comment on the individual nurse as well as the LCNS role, and be constrained in being open and honest. This was considered by those conducting interviews and the interview schedule questions constructed to minimise this risk. The emphasis in the interview schedule was on their understanding of the nature of LCNS role. They were not invited to make a judgement of the quality of the LCNS practice.

\section{Comparison with other data}

There is growing interest in the value of the LCNS and their contribution to patient experience and positive outcomes of care ${ }^{6-9}{ }^{12-15}$ Findings have been reinforced by other publications since the completion of this study, for example, those highlighting nurse specialist skills in patient assessment and the advanced level of practice and ability to navigate NHS organisations. ${ }^{17}$

This study also echoes findings from questionnaire-led research that identifies most LCNSs provide care across the patient pathway from diagnosis onwards, ${ }^{14}$ and that symptom management is a highly ranked activity for
LCNSs. ${ }^{15}$ Our findings also chime with previous surveys by the Roy Castle Foundation and NLCFN indicating that the LCNS is likely to improve access to treatment if they see the patient early in the pathway, actively manage patient symptoms and are independent prescribers. ${ }^{6}$ This is especially the case for chemotherapy. ${ }^{9}$ Our findings reinforce this evidence and expand our understanding of how LCNS may open doors to treatment.

In addition, this study shows how holistic assessment, patient focused case management and provision of psychological support can contribute to treatment access and uptake. Worryingly, Leary et $a l^{14}$ reveal that more LCNSs are increasingly unable to undertake these aspects of care. Reasons include caseload size and pressures, and having to take roistered shifts on wards because of staff shortages.

\section{Implications of our findings}

The findings here clearly demonstrate the contribution LCNSs can make to treatment access and uptake. If UK lung cancer patient survival rates are to improve in line with comparable countries, MDTs need to employ every advantage. This includes promoting the LCNS role in line with national evidence-based guidance and recommendations ${ }^{62526}$ In financially constrained environments, it may be tempting to see the LCNS as an expensive resource, and therefore vulnerable to cuts. ${ }^{6}{ }^{14} 17$ However, this study demonstrates how integral the role is to efficient and quality MDT clinical care delivery.

There is an urgent need for an economic evaluation of the impact of roles such as the LCNS. Robust costbenefit and cost effectiveness studies would be a challenge but are essential.

Acknowledgements The authors are extremely grateful to all the participants, especially those who were case studies. The authors thank the participants for their generosity in sharing their time, views and experiences.

Contributors AMT and AM originated the idea for the study. AMT and AM designed the study in collaboration with JR, DB and JW. JR conducted all the data collection and analysis alongside either AMT or AM. DB and JW supported recruitment. All authors commented critically on the analysis and drafts of the paper

Funding This research was supported by funding from the General Nursing Council Trust for England and Wales (GNCT), National Lung Cancer Forum for Nurses (NLCFN).

\section{Competing interests None declared.}

Ethics approval NHS Research Governance approval was obtained from all the study sites. Ethical approval was obtained by Sheffield Hallam University, Faculty of Health and Wellbeing Research Ethics Committee.

Provenance and peer review Not commissioned; externally peer reviewed.

Data sharing statement Primary anonymised data is available on request to corresponding author.

Open Access This is an Open Access article distributed in accordance with the Creative Commons Attribution Non Commercial (CC BY-NC 4.0) license, which permits others to distribute, remix, adapt, build upon this work noncommercially, and license their derivative works on different terms, provided the original work is properly cited and the use is non-commercial. See: http:// creativecommons.org/licenses/by-nc/4.0/ 


\section{REFERENCES}

1. Cancer Research UK. Cancer incidence statistics. 2014. http://www. cancerresearchuk.org/cancer-info/cancerstats/incidence/ (accessed Aug 2014).

2. Walters S, Maringe C, Coleman MP, et al., ICBP Module 1 Working Group. Lung cancer survival and stage at diagnosis in Australia, Canada, Denmark, Norway, Sweden and the UK: a population-based study, 2004-2007. Thorax 2013;68:551-64.

3. Health and Social Care Information Centre National Lung Cancer Audit Report 2013. Report for the audit period. 2012. http://www. hscic.gov.uk/catalogue/PUB12719/clin-audi-supp-prog-lung-nlca2013-rep.pdf

4. Cancer Research UK. Lung cancer survival rates improving. 2013. http://www.cancerresearchuk.org/about-us/cancer-news/news-report/ lung-cancer-survival-rates-improving (accessed Aug 2014).

5. Russell GK, Jimenez S, Martin L, et al. A multicentre randomised controlled trial of reciprocal lung cancer peer review and supported quality improvement: results from the improving lung cancer outcomes project. Br J Cancer 2014;110:1936-42.

6. Roy Castle Lung Cancer Foundation. Understanding the value of lung cancer nurse specialists. 2013: http://www.roycastle.org/ Resources/Roy\%20Castle/Documents/PDF/

UnderstandTheValueOfLungCancerNurseSpecialists_V03.pdf (accessed Aug 2014).

7. Ford S. Lung cancer patients twice as likely to get treatment if they see a nurse. Nursing Times. 2011. http://www.nursingtimes.net/ nursing-practice/clinical-specialisms/cancer/lung-cancer-patientstwice-as-likely-to-get-treatment-if-they-see-a-nurse/5038833.article (accessed Aug 2014).

8. [No authors listed]. Specialist nurses and lung cancer. Lancet 2011;377:1892.

9. Beckett P, Woolhouse I, Stanley R, et al. Nurse specialist input is independently associated with anti-cancer treatment in lung cancer. Thorax 2011;66:(Suppl 4)A42-3.

10. Health and Social Care Information Centre National Lung Cancer Audit Report 2012. Report for the audit period. 2011. http://www. hscic.gov.uk/catalogue/PUB09183/clin-audi-supp-prog-lung-nlca-lap2012-rep.pdf

11. Health and Social Care Information Centre National Lung Cancer Audit Report 2011. Report for the audit period. 2010. http://www. hscic.gov.uk/catalogue/PUB02676/clin-audi-supp-prog-lung-cancnlca-2011-rep.pdf

12. National Cancer Action Team. Quality in nursing. Excellence in nursing: The contribution of the clinical nurse specialist. 2010. http://www.cancernurse.eu/documents/ NCATMacmillanReportOctober2010.pdf (accessed Aug 2014).
13. Leary A, Bell N, Darlison L, et al. An analysis of lung cancer clinical nurse specialist workload and value. Cancer Nurs Pract 2008;7:29-33.

14. Leary A, White J, Yarnell L. The work left undone. Understanding the challenge of providing holistic lung cancer nursing care in the UK. Eur J Oncol Nurs 2014;18:23-8.

15. McPhelim J. A review of the role of the lung cancer nurse specialist. Cancer Nurs Prac 2009;8:30-2.

16. McDonnell A, Gerrish K, Kirshbaum MN, et al. The perceived impact of advanced practice nurses (APNs) on promoting evidence-based practice amongst frontline nurses: findings from a collective case study. J Res Nurs 2013;18:368-83.

17. Read C. Time for some advanced thinking? The benefits of specialist nurses. A Health Service Journal Supplement. 2015. http://www.hsj.co.uk/Journals/2015/02/25/f/c/y/

HSJ-Workforce-Supplement-150227.pdf (accessed Mar 2015).

18. Stake RE. Case study analysis. New York: The Guilford Press 2006.

19. Thomas G. How to do your case study. London: Sage Publications Ltd, 2010.

20. Tod AM, McDonnell A, Redman J. Opening doors to treatment: exploring the impact of lung cancer specialist nurses on access to anti-cancer treatment: an exploratory case study. 2013. http://www. shu.ac.uk/research/hsc/sites/shu.ac.uk/files/REVISED\%20FINAL\% 20DRAFT\%20GNC\%20T\%20LCNS\%207\%203\%2014\%20(2).pdf (accessed Augt 2014).

21. Rich AL, Tata LJ, Free CM, et al. Inequalities in outcomes for non-small cell lung cancer: the influence of clinical characteristics and features of the local lung cancer service. Thorax 2011;66:1078-84.

22. Ritchie J. Lewis J. Qualitative research practice. London: Sage, 1994

23. Ritchie J, Spencer L. Qualitative data analysis for applied policy research. In: Bryman A, Burgess RG, eds. Analysing qualitative research. London: Routeledge, 1994:173-97.

24. McDonnell A, Smith C, Goodwin E, et al. An evaluation of the implementation of Advanced Nurse Practitioner (ANP) roles at Barnsley Hospital NHS Foundation Trust. Unpublished final report. Sheffield: Sheffield Hallam University, 2013.

25. National Institute for Health and Clinical Excellence Lung cancer The diagnosis and treatment of lung cancer. Clinical Guideance 121. 2011. http://www.nice.org.uk/guidance/cg121/resources/ guidance-lung-cancer-pdf (accessed Aug 2014).

26. United Kingdom Lung Cancer Coalition. The Dream MDT. 2013. http://www.uklcc.org.uk/files/pdf/UKLCC___Putting_patients_first_Understanding_what_matters_most_to_lung_cancer_patients_and_ carers.pdf (accessed Aug 2014). 